\title{
Value of Information Web Application
}

\author{
by John T Richardson
}




\section{NOTICES}

\section{Disclaimers}

The findings in this report are not to be construed as an official Department of the Army position unless so designated by other authorized documents.

Citation of manufacturer's or trade names does not constitute an official endorsement or approval of the use thereof.

Destroy this report when it is no longer needed. Do not return it to the originator. 


\title{
Army Research Laboratory
}

Aberdeen Proving Ground, MD 21005-5067

ARL-TN-0669

April 2015

\section{Value of Information Web Application}

\author{
John T Richardson \\ Computational and Information Sciences Directorate, ARL
}




\section{REPORT DOCUMENTATION PAGE}

Public reporting burden for this collection of information is estimated to average 1 hour per response, including the time for reviewing instructions, searching existing data sources, gathering and maintaining the data needed, and completing and reviewing the collection information. Send comments regarding this burden estimate or any other aspect of this collection of information, including suggestions for reducing the burden, to Department of Defense, Washington Headquarters Services, Directorate for Information Operations and Reports (0704-0188), 1215 Jefferson Davis Highway, Suite 1204, Arlington, VA 22202-4302. Respondents should be aware that notwithstanding any other provision of law, no person shall be subject to any penalty for failing to comply with a collection of information if it does not display a currently valid OMB control number.

PLEASE DO NOT RETURN YOUR FORM TO THE ABOVE ADDRESS.

\begin{tabular}{|l|l|l}
\hline 1. REPORT DATE $(D D-M M-Y Y Y Y)$ & 2. REPORT TYPE & 3. DATES COVERED (FrOm - To)
\end{tabular}

April 2015 Final

4. TITLE AND SUBTITLE

October 2013-September 2014

Value of Information Web Application 5a. CONTRACT NUMBER

\begin{tabular}{|c|c|}
\hline che & 5b. GRANT NUMBER \\
\hline \multirow{2}{*}{$\begin{array}{l}\text { 6. AUTHOR(S) } \\
\text { John T Richardson }\end{array}$} & \\
\hline & 5e. TASK NUMBER \\
\hline $\begin{array}{l}\text { 7. PERFORMING ORGANIZATION NAME(S) AND ADDRESS(ES) } \\
\text { US Army Research Laboratory } \\
\text { ATTN: RDRL-CII-T } \\
\text { Aberdeen Proving Ground, MD } 21005-5067\end{array}$ & $\begin{array}{l}\text { 8. PERFORMING ORGANIZATION } \\
\text { REPORT NUMBER } \\
\text { ARL-TN-0669 }\end{array}$ \\
\hline
\end{tabular}

Approved for public release; distribution is unlimited.

\section{SUPPLEMENTARY NOTES}

\section{ABSTRACT}

This report discusses the design and use of a value of information web application. The application allows US Army Research Laboratory researchers to collect statistically relevant data from subject matter experts in the field of intelligence analysis. The data will be used in the future to validate the current Fuzzy Associate Memory Value of Information prototype.

\section{SUBJECT TERMS}

value of information, fuzzy associate memory, web application, intelligence analysis, statistics

\begin{tabular}{|l|l|l|l|c|l|}
\hline \multicolumn{2}{|l|}{ 16. SECURITY CLASSIFICATION OF: } & $\begin{array}{l}\text { 17. LIMITATION } \\
\text { OF ABSTRACT }\end{array}$ & $\begin{array}{l}\text { 18. NUMBER } \\
\text { OF PAGES }\end{array}$ & $\begin{array}{l}\text { 19a. NAME OF RESPONSIBLE PERSON } \\
\text { John T RichardsOn }\end{array}$ \\
\cline { 1 - 2 } $\begin{array}{l}\text { a. REPORT } \\
\text { Unclassified }\end{array}$ & $\begin{array}{l}\text { b. ABSTRACT } \\
\text { Unclassified }\end{array}$ & $\begin{array}{l}\text { c. THIS PAGE } \\
\text { Unclassified }\end{array}$ & UU & 28 & $\begin{array}{l}\text { 19b. TELEPHONE NUMBER (Include area code) } \\
410-278-4143\end{array}$ \\
\hline
\end{tabular}




\section{Contents}

$\begin{array}{ll}\text { Contents } & \text { iii }\end{array}$

List of Figures $\quad$ iv

$\begin{array}{ll}\text { 1. Introduction } & 1\end{array}$

2. Value of Information Web Application $\quad 1$

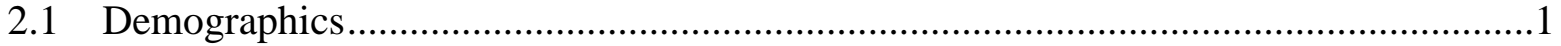

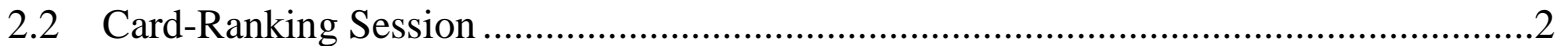

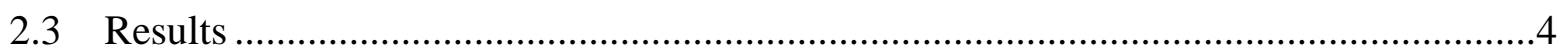

3. Software Development 5

4. Conclusions $\quad 6$

$\begin{array}{lll}\text { 5. } & \text { References } & 7\end{array}$

$\begin{array}{lr}\text { Appendix. Value of Information Source Code } & 9\end{array}$

$\begin{array}{ll}\text { Distribution List } & 22\end{array}$ 


\section{List of Figures}

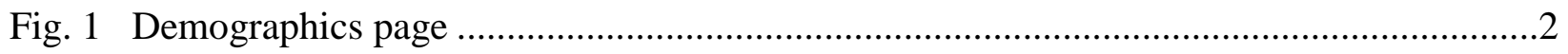

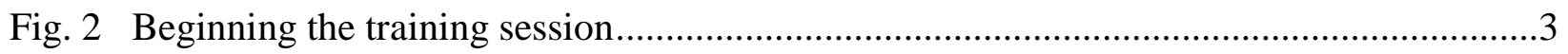

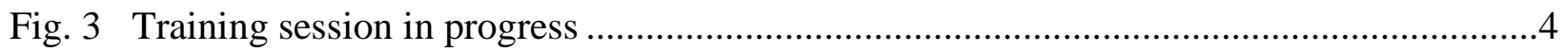

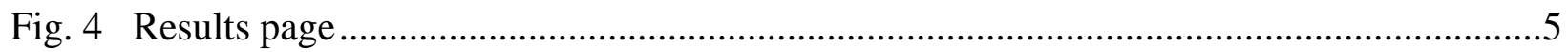




\section{Introduction}

Modern military operations use information from a large number of sources resulting in potential data overload. To process these data, it is necessary to rank the data in a manner such that the most valuable information can be analyzed first. This ranking metric is termed the value of information (VoI) and its calculation is a current area of research within the US Army Research Laboratory (ARL). Researchers at ARL have designed and experimented with a fuzzy associate memory (FAM) approach to calculating VoI. ${ }^{1}$ They identified the need to validate the system with a statistically relevant experiment to compare the ranking of information by subject matter experts (SMEs) against the FAM prototype. This report discusses a web-based tool designed for collecting rank data from SME users that can be used in future statistical analysis. The following sections will discuss the use and development of the VoI web application.

\section{Value of Information Web Application}

The goal of the VoI web application is to present an SME with several scenarios, represented by a hand of cards representing data of unranked value. The web application will record the order the SME ranks the cards and how long the process takes to complete. These data will be used by ARL researchers to refine their understanding of VoI attributes (source reliable, information content, and latency).

The VoI web application emulates many features of a previously created tool developed in Visual Basic by Dr Timothy Hanratty. The original tool required an executable to be deployed on a computer running a compatible version of the Windows operating system. This limited the amount of users able to access the tool. As a result, the VoI web application was built to allow the tool to be accessed from the Internet via a web browser. The following sections describe the web application's user interface.

\subsection{Demographics}

The initial page that the user encounters when accessing the VoI web application is Demographics (Fig. 1). On this page, the user can provide optional information that is recorded with their results. The user can fill in all, some, or none of the information and then click the Submit Demographics button to continue to the card-ranking sessions. 


\section{Demographics}

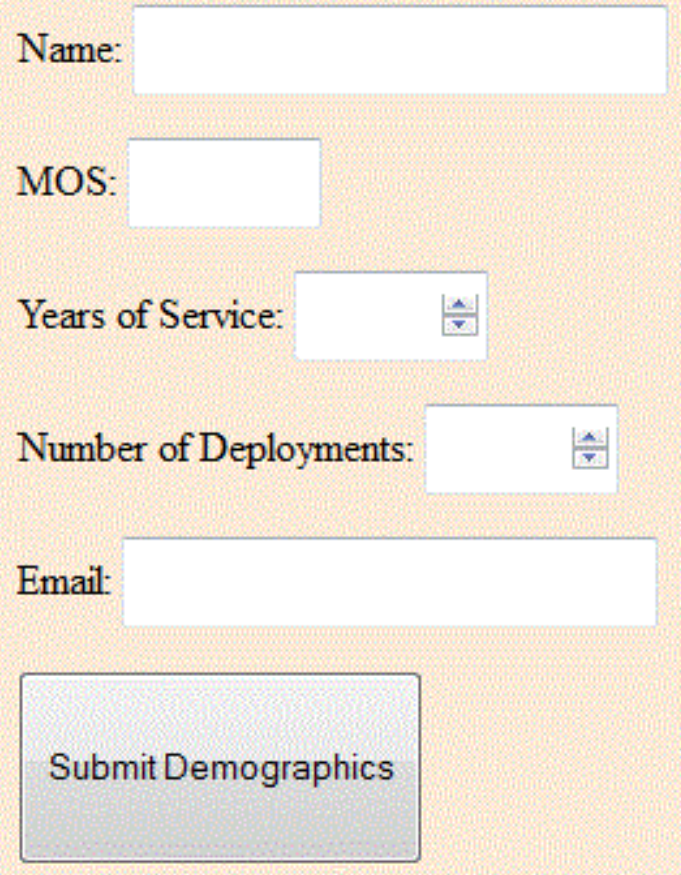

Fig. 1 Demographics page

\subsection{Card-Ranking Session}

On the Card-Ranking Session page, the SME uses personal judgment to rank a series of cards, each card representing a single piece of information. The SME ranks each card based upon 3 attributes: source reliable, information content, and latency. The cards are divided into 4 decks: training, tactical, strategic, and operational. Each deck, other than training, consists of 40 hands of 5 or 7 cards. The training deck has 3 hands as its only use is to familiarize the user with the system. During the sessions, the web application records the rank the user chooses for each hand and the amount of time taken to perform the ranking. These data will be used for future statistical analysis that is not discussed in this report.

The 4 decks are accessed in the order training, tactical, strategic, and operational. When the user is ready to view the first hand in a deck, the user presses the Begin \$ Session button, where \$ stands for the name of the next deck to be played. For example, the button initially reads Begin Training Session, since that is the first deck a user plays. Once a hand is dealt, this button is grayed out until all the hands in the deck are finished. The red text at the top of the page always informs the user which deck they are currently playing. Figure 2 displays the session page at the point when a user is dealt the first hand from the training deck. 


\section{Training Session}

\section{Given Data Set}

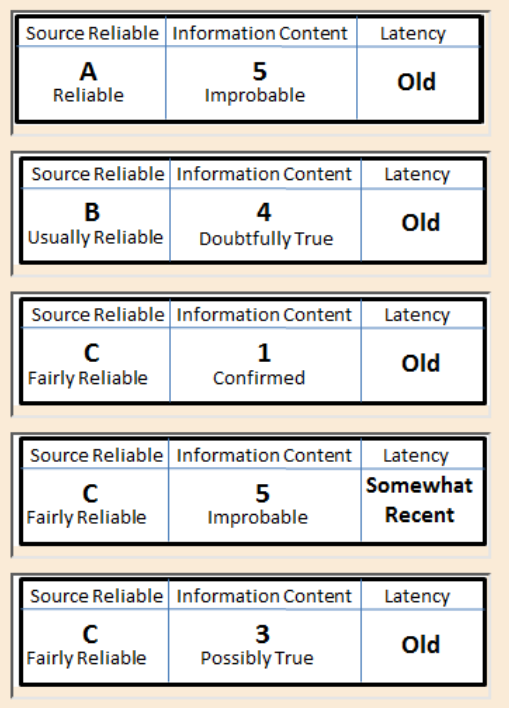

Ranked Data Set

Highest VoI

2nd Highest

3rd Highest

4th Highest

Lowest VoI
Instructions: Please rank the Information Cards in the Ieft Column into the Spaces in the column into the spaces in the Right colum by dragging and

Begin Training Session

Enter Scores

Reset

Exit

Count: 2

Fig. 2 Beginning the training session

To play the hand, the user considers the attributes on each card and then drags and drops the cards from the Given Data Set column to the Ranked Data Set column to arrange the cards from highest to lowest VoI (Fig. 3). During this ranking process, the user may return all the cards to their original state in the Given Data Set column by pressing the Reset button. This allows the user to restart the ranking process but does not restart the timer that is recording how long it takes the user to evaluate the hand. When the user is satisfied with the ranking of the hand, the user presses the Enter Scores button. This action finishes the data collection on the current hand and deals a new hand (unless the deck is empty). Consequently, the Count box will increment by one to indicate the current hand in play. If the deck is empty, the web application sets up the next deck for the user or sends the user to the Results page if all decks have been played. The user can also exit to the Results page at anytime by pressing the Exit button. 


\section{Training Session}

Given Data Set
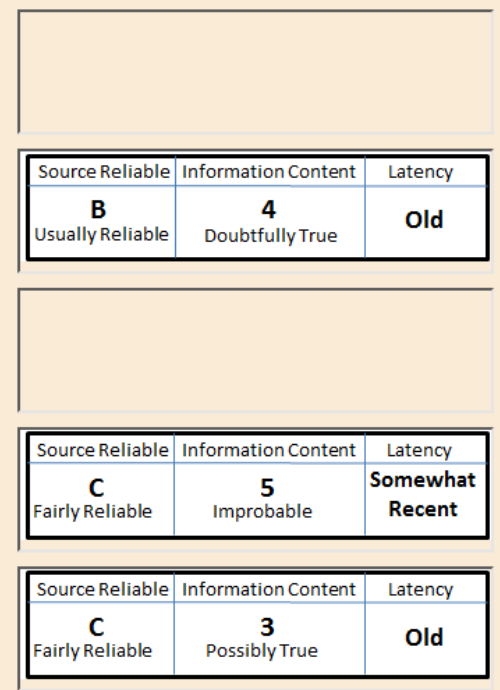

Ranked Data Set

Highest VoI

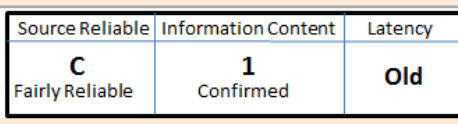

2nd Highest

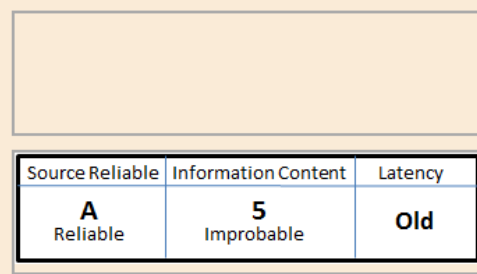

4th Highest

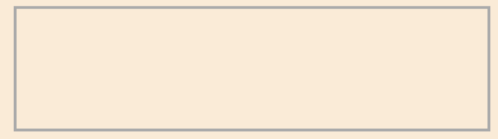

Lowest VoI
Instructions: Please rank th Information Cards in the Lef Column into the Spaces in the Right Column by dragging and the Cards into the appropriate slot.

Begin Training Session

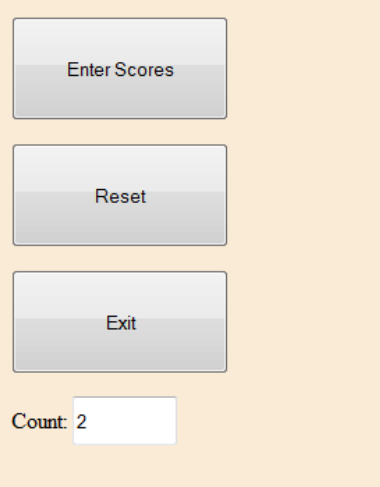

Fig. 3 Training session in progress

\subsection{Results}

The Results page shows the user the session data (Fig. 4). It includes the deck, the original and ranked card order for each hand, and the amount of time, rounded to the nearest second, that it took to rank the hand. From this page, the user may also review and update their demographic information by pressing the Edit Demographics button. Finally, the user may send the data to the researchers by pressing the Submit Results button. The user receives a confirmation that the submittal was successful or not successful. This ends the users' interaction with the web application. 


\section{Results}
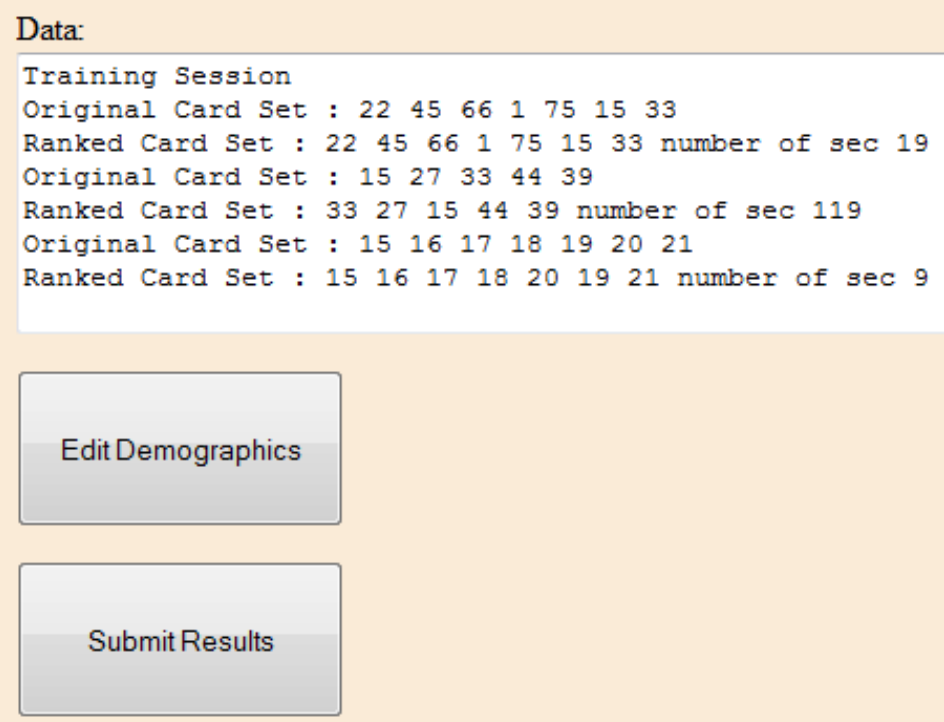

Fig. 4 Results page

\section{Software Development}

The VoI web application was designed and written by ARL. The pages viewed by the client, and discussed in the previous sections, were developed using HTML 5 and JavaScript (see the Appendix for source code). The server side script for processing the submitted results was written using PHP: Hypertext Preprocessor (PHP). The client side of the application was tested on ARL standard computers and functioned properly only when using the Firefox web browser on those computers (Internet Explorer was not viable due to unchangeable user settings). During testing, the web application was hosted successfully on the Tactical Information Fusion Integrated Digital Virtual Research Network enclave using both Apache httpd and Microsoft IIS web server software. 


\section{Conclusions}

The development of the VoI web tool delivers an Internet-accessible web page with the ability to collect data for the statistical refinement of the VoI parameters, which may be detailed in future reports. The final step in deployment is to identify a host server for the web page that is reachable by relevant SMEs. Furthermore, the server side data processing should be modified to adapt to data collection requirements. The current solution stores the results in text files because of the small number of users. Interaction with a database may be required if usage ever increases significantly. 


\section{References}

1. Hammell RJ II, Hanratty T, Heilman E. Capturing the value of information in complex military environments: A fuzzy-based approach. Proceedings of the 2012 IEEE International Conference on Fuzzy Systems; 2012 June 10-15; Brisbane, Australia. Piscataway (NJ): IEEE c2012. 
INTENTIONALLY LEFT BLANK. 


\section{Appendix. Value of Information Source Code}

This appendix appears in its original form, without editorial change. 
<!DOCTYPE html PUBLIC "-//W3C//DTD XHTML 1.1//EN"

"http://www.w3.org/TR/xhtml11/DTD/xhtml11.dtd">

$<$ html xmlns='http://www.w3.org/1999/xhtml'>

$<$ head >

$<$ style>

\#div1, \#div2, \#div3, \#div4, \#div5, \#div6, \#div7

\{width:338px; height:86px;margin:10px;border:2px inset \#aaaaaa;

\#div8, \#div9, \#div10, \#div11, \#div12, \#div13, \#div14

\{width:338px; height:86px; margin:10px;border:2px solid \#aaaaaa;

$</$ style $>$

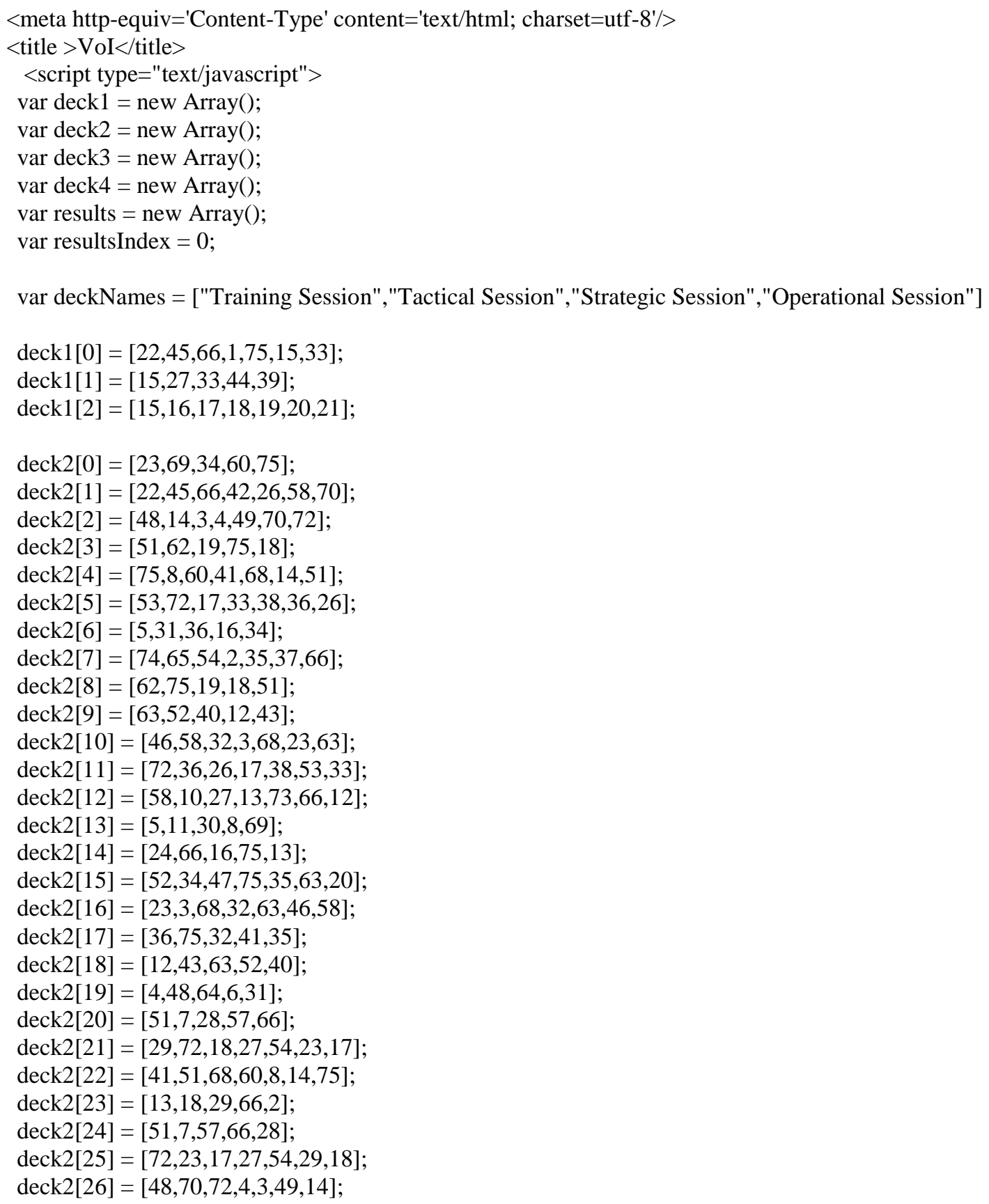




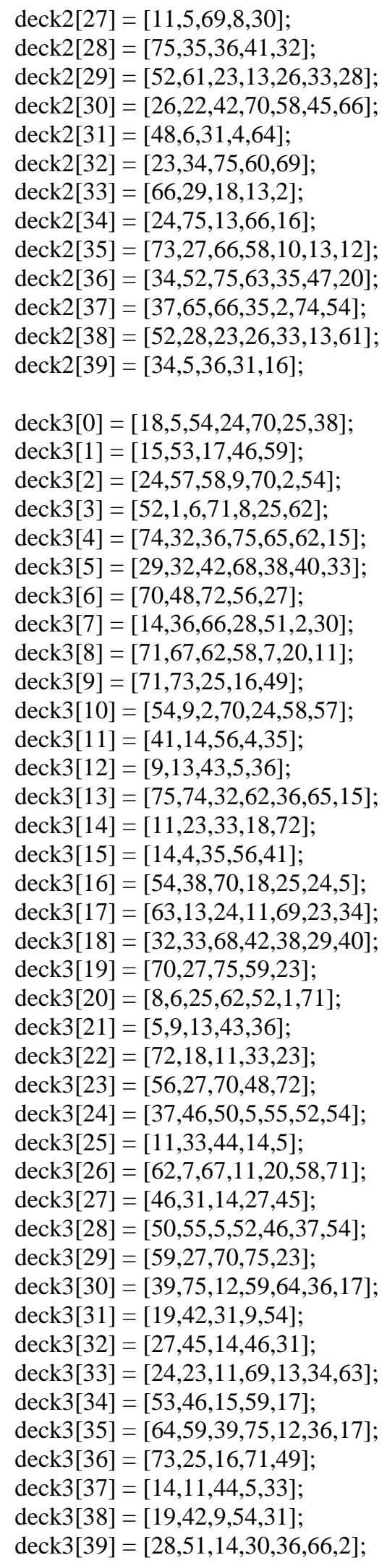




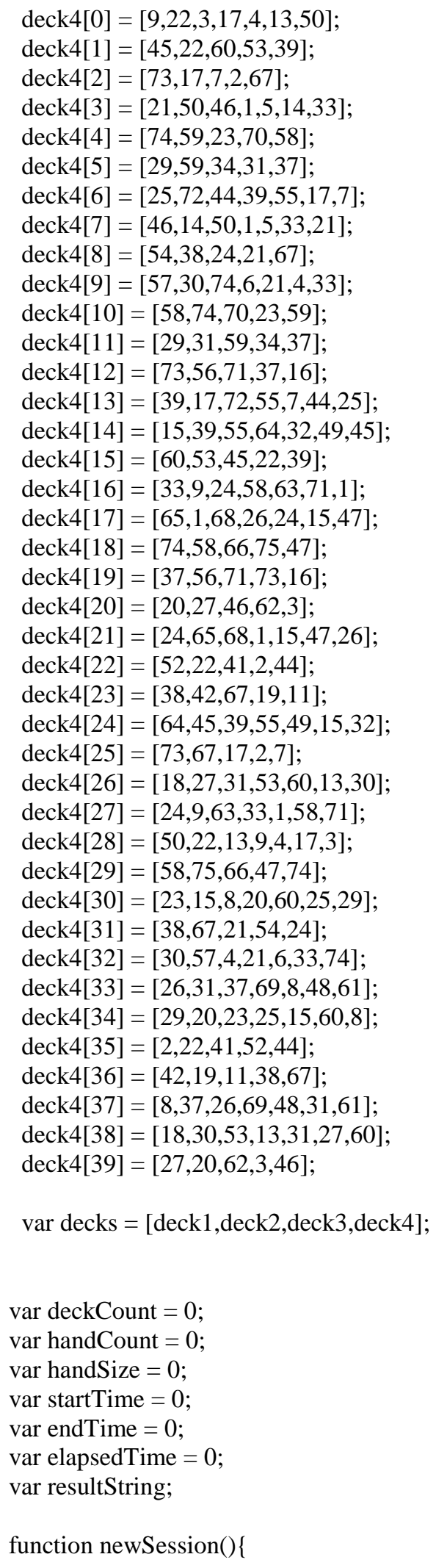




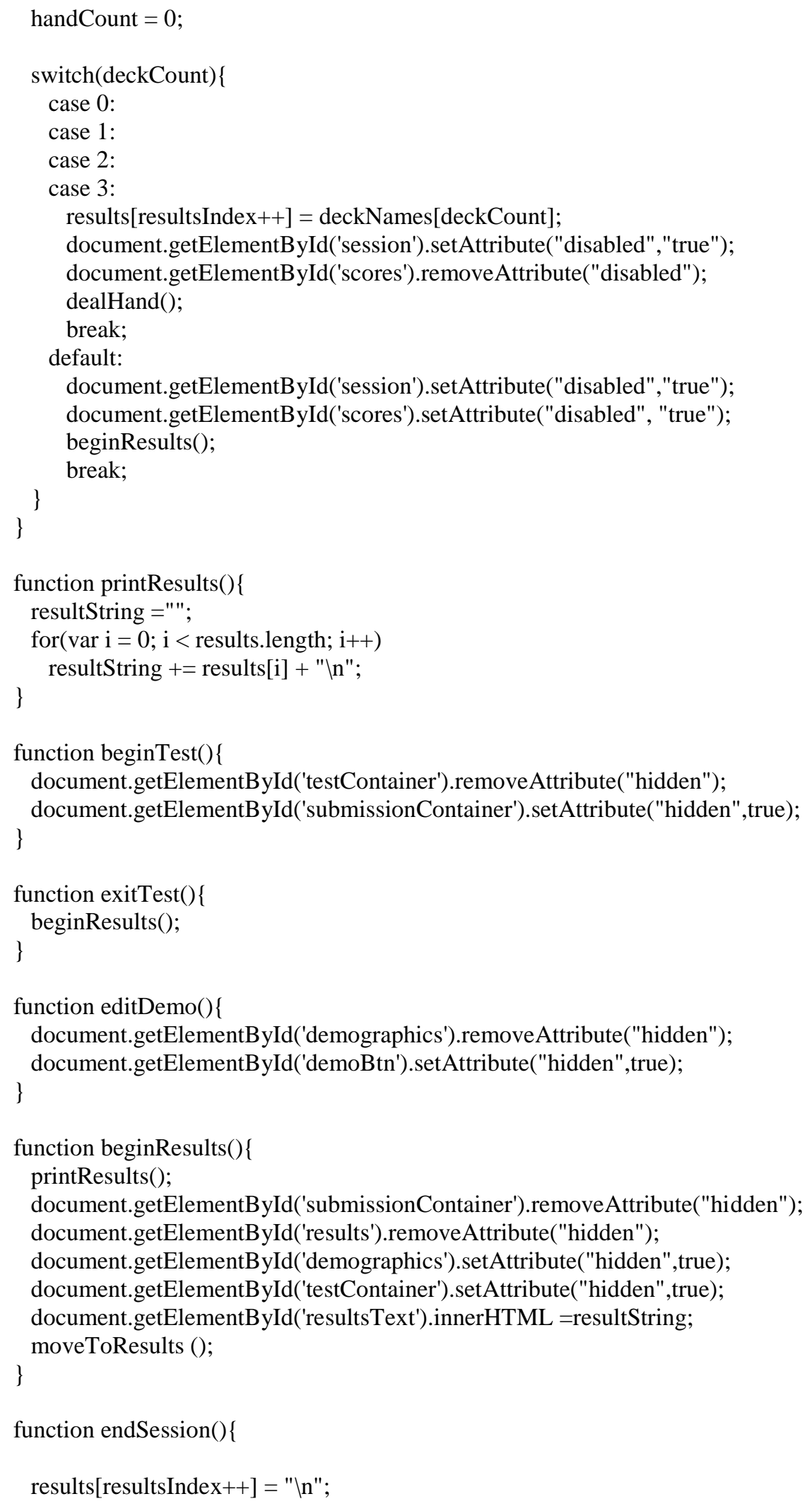




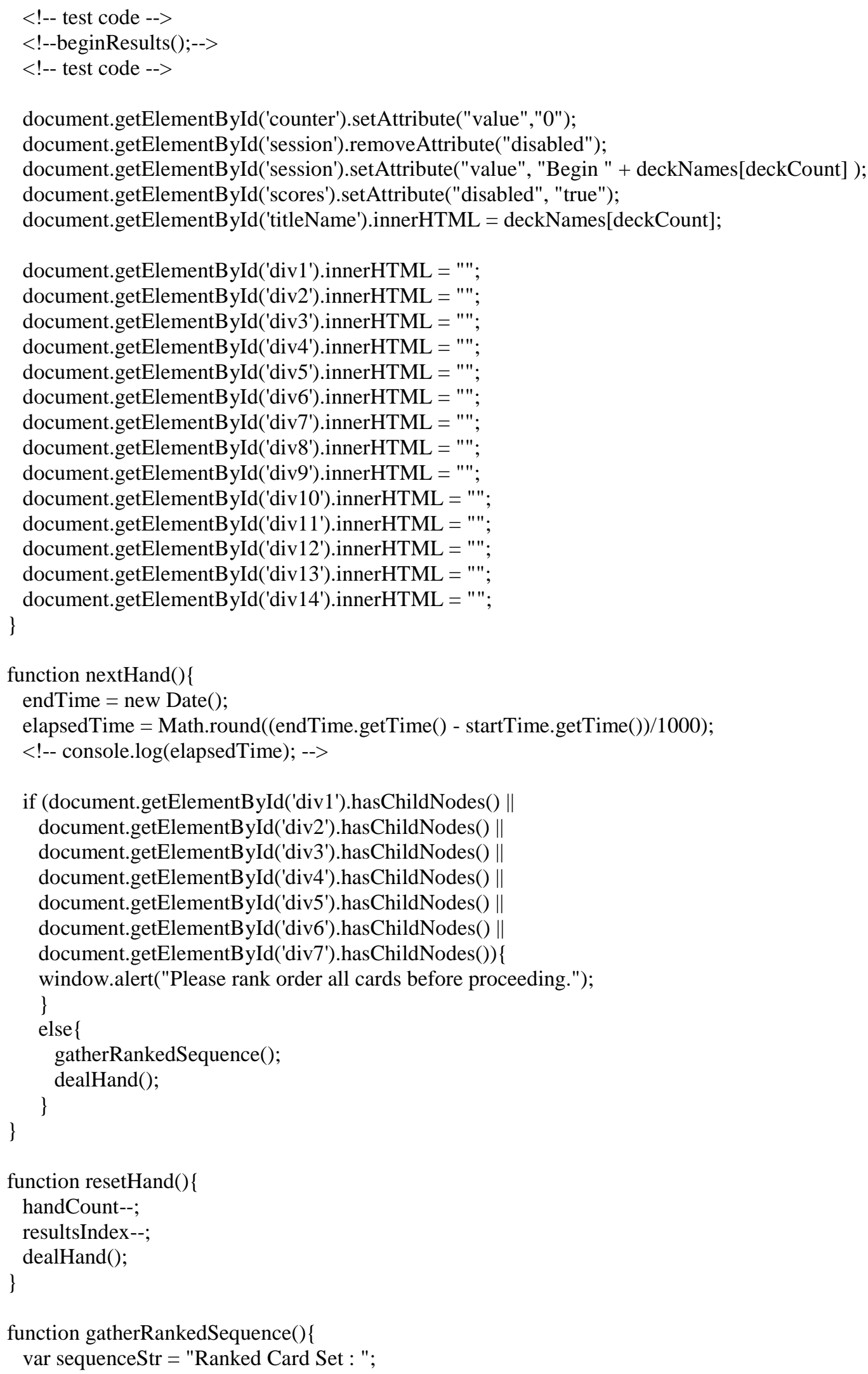




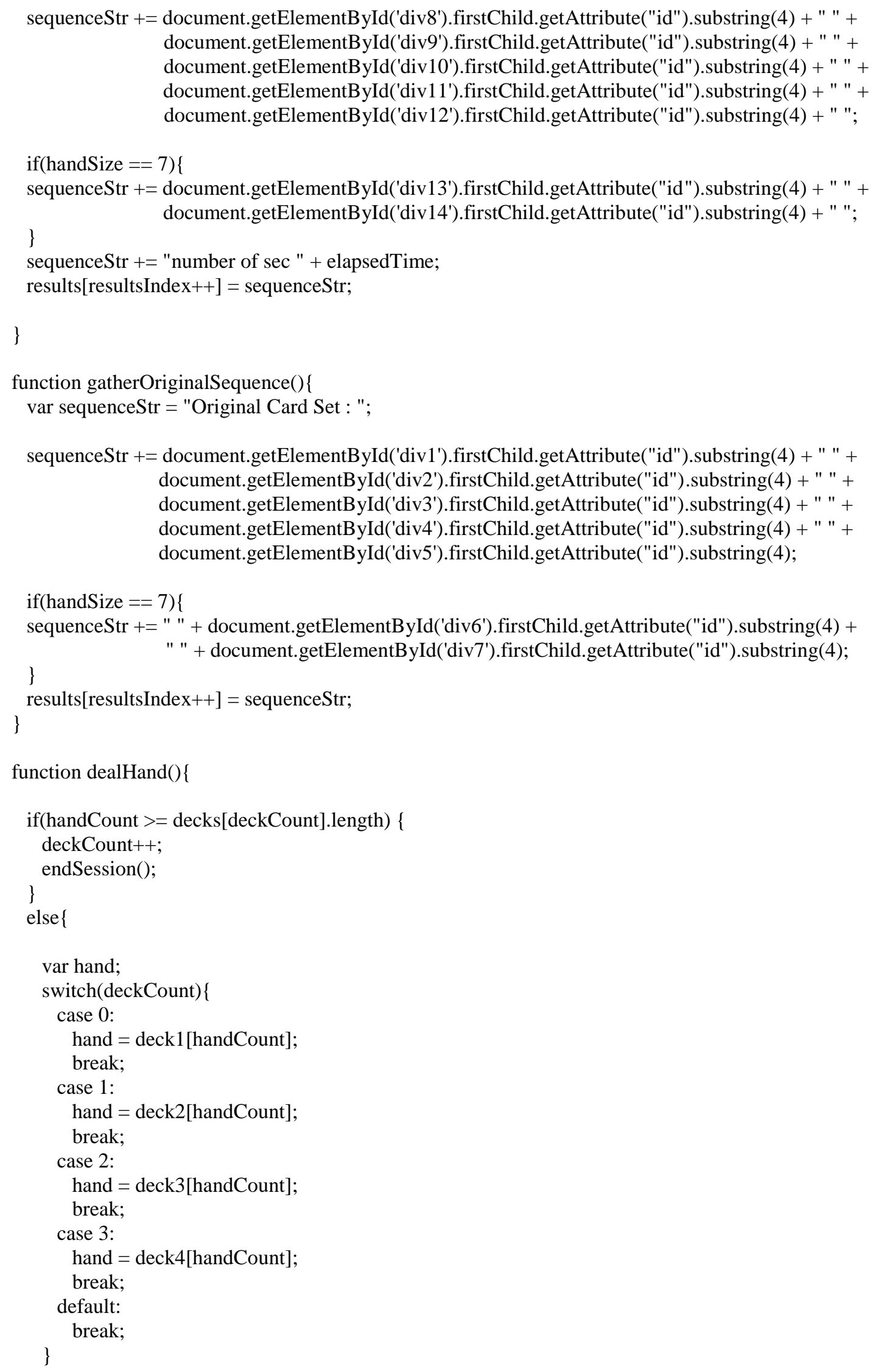


handSize $=$ hand.length;

if $($ handSize $==7)\{$

document.getElementById('div1').innerHTML = "<img id=l"card" + hand[0] + "।" draggable=|"truel" ondragstart=l"drag(event)।" src=l"images/card" + hand[0] + ".png|">";

document.getElementById('div2').innerHTML = "<img id=l"card" + hand[1] + "l" draggable=l"truel"

ondragstart=l"drag(event)।" src=l"images/card" + hand[1] + ".png|">";

document.getElementById('div3').innerHTML = "<img id=|"card" + hand[2] + "।" draggable=|"truel"

ondragstart=l"drag(event)।" src=l"images/card" + hand[2] + ".png|">";

document.getElementById('div4').innerHTML = "<img id=l"card" + hand[3] + "l" draggable=l"truel"

ondragstart=l"drag(event)\" src=l"images/card" + hand[3] + ".pngl">";

document.getElementById('div5').innerHTML = "<img id=l"card" + hand[4] + "l" draggable=l"truel"

ondragstart=|"drag(event)।" src=|"images/card" + hand[4] + ".png|">";

document.getElementById('div6').innerHTML = "<img id=l"card" + hand[5] + "।" draggable=l"truel"

ondragstart=l"drag(event)\" src=l"images/card" + hand[5] + ".pngl">";

document.getElementById('div7').innerHTML = "<img id=।"card" + hand[6] + "।" draggable=|"truel"

ondragstart=l"drag(event)\" src=|"images/card" + hand[6] + ".png|">";

document.getElementById('div6').removeAttribute("hidden");

document.getElementById('div7').removeAttribute("hidden");

document.getElementById('label5').innerHTML = "5th Lowest";

document.getElementById('label6').removeAttribute("hidden");

document.getElementById('label7').removeAttribute("hidden");

document.getElementById('div8').innerHTML = "";

document.getElementById('div9').innerHTML = "";

document.getElementById('div10').innerHTML = "";

document.getElementById('div11').innerHTML = "";

document.getElementById('div12').innerHTML = "';

document.getElementById('div13').innerHTML = "";

document.getElementById('div14').innerHTML = "";

document.getElementById('div13').removeAttribute("hidden");

document.getElementById('div14').removeAttribute("hidden");

\}

else\{

document.getElementById('div1').innerHTML = "<img id=।"card" + hand[0] + "।" draggable=|"truel" ondragstart=|"drag(event)\" src=|"images/card" + hand[0] + ".png|">";

document.getElementById('div2').innerHTML = "<img id=l"card" + hand[1] + "।" draggable=|"truel" ondragstart=l"drag(event)।" src=l"images/card" + hand[1] + ".png|">";

document.getElementById('div3').innerHTML ="<img id=|"card" + hand[2] + "।" draggable=|"truel"

ondragstart=l"drag(event)।" src=l"images/card" + hand[2] + ".pngl">";

document.getElementById('div4').innerHTML = "<img id=l"card" + hand[3] + "।" draggable=l"truel"

ondragstart=|"drag(event)\" src=|"images/card" + hand[3] + ".png|">";

document.getElementById('div5').innerHTML = "<img id=l"card" + hand[4] + "l" draggable=l"truel"

ondragstart=l"drag(event)।" src=l"images/card" + hand[4] + ".png|">";

document.getElementById('div6').innerHTML = "";

document.getElementById('div7').innerHTML = "";

document.getElementById('div6').setAttribute("hidden","true");

document.getElementById('div7').setAttribute("hidden","true");

document.getElementById('label5').innerHTML = "Lowest VoI";

document.getElementById('label6').setAttribute("hidden","true");

document.getElementById('label7').setAttribute("hidden","true");

document.getElementById('div8').innerHTML = "";

document.getElementById('div9').innerHTML = "';

document.getElementById('div10').innerHTML = "";

document.getElementById('div11').innerHTML = "';

document.getElementById('div12').innerHTML = "";

document.getElementById('div13').setAttribute("hidden","true"); 


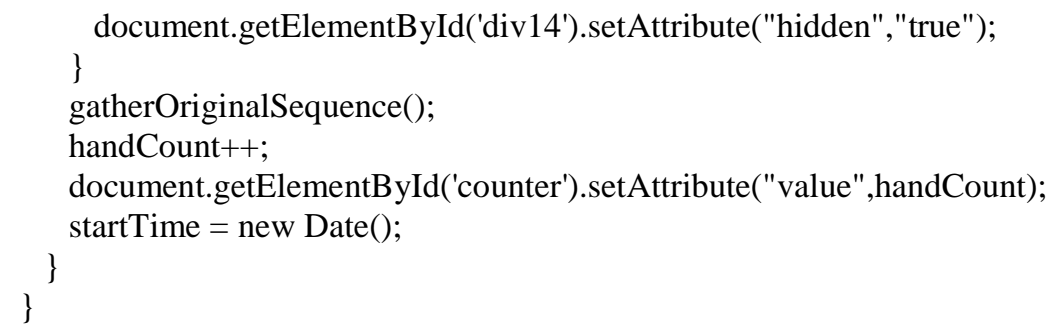


$<\mathrm{h} 3 \mathrm{id}="$ label3" style="text-align:center;width:150px; height:86px; margin:12px;">3rd Highest $</ \mathrm{h} 3>$ $<$ h3 id="label4" style="text-align:center;width:150px; height:86px; margin:12px;">4th Highest $</ \mathrm{h} 3>$ $<\mathrm{h} 3$ id="label5" style="text-align:center;width:150px; height:86px; margin:12px;" $>$ 5th Highest $</ \mathrm{h} 3>$ $<$ h3 id="label6" style="text-align:center;width:150px; height:86px; margin:12px;">6th Highest $</ \mathrm{h} 3>$ $<\mathrm{h} 3 \mathrm{id}="$ label7" style="text-align:center;width:150px; height:86px; margin:12px;">Lowest $\mathrm{VoI}</ \mathrm{h} 3>$ $</$ div $>$

$<$ div id="rankedData" style="width:362px;float:left;"> $<$ h1 style="text-align:center;" >Ranked Data Set $</$ h1 $>$ $<$ div id="div8" ondrop="drop(event)" ondragover="allowDrop(event)" $></$ div $>$ $<$ div id="div9" ondrop="drop(event)" ondragover="allowDrop(event)" $></$ div $>$ $<$ div id="div10" ondrop="drop(event)" ondragover="allowDrop(event)" $></$ div $>$ $<\operatorname{div}$ id="div11" ondrop="drop(event)" ondragover="allowDrop(event)" $></$ div $>$ $<\operatorname{div}$ id="div12" ondrop="drop(event)" ondragover="allowDrop(event)" $></$ div $\rangle$ $<$ div id="div13" ondrop="drop(event)" ondragover="allowDrop(event)" $></$ div $\rangle$ $<$ div id="div14" ondrop="drop(event)" ondragover="allowDrop(event)" $>\langle/$ div $\rangle$ $</$ div $>$

<div id="controls" style="width:362px;float:left;">

<textarea style="height: $115 \mathrm{px}$; width: 258px; resize: none" readonly>Instructions: Please rank the Information Cards in the Left Column into the Spaces in the Right Column by dragging and dropping the Cards into the appropriate slot.

$</$ textarea $\rangle$

$<\mathrm{p}>$

<input type='button' id='session' autocomplete="off" style="height: 75px; width: 156px" value='Begin

Training Session' onclick="newSession();" />

$</ \mathrm{p}\rangle$

$\langle\mathrm{p}\rangle$

<input disabled type='button' id='scores' autocomplete="off" style="height: 75px; width: 156px"

value='Enter Scores' onclick="nextHand();" />

$\langle/ \mathrm{p}\rangle$

$\langle\mathrm{p}\rangle$

$<$ input type='button' id='reset' autocomplete="off" style="height: 75px; width: 156px" value='Reset' onclick="resetHand();" $/>$

$\langle/ \mathrm{p}\rangle$

$\langle\mathrm{p}\rangle$

$<$ input type='button' id='exit' autocomplete="off" style="height: 75px; width: 156px" value='Exit' onclick="exitTest();" />

$</ \mathrm{p}\rangle$

$\langle\mathrm{p}\rangle$

Count: <input type='text' autocomplete="off" id="counter" style="height: 29px; width: 69px" value='0' readonly/>

$</ \mathrm{p}\rangle$

$\langle/$ div $\rangle$

$</$ div $>$

<div style="clear:both;margin-left:auto;margin-right:auto;width:720px;" id="submissionContainer" >

$<$ form id='form' action='voi_results.php' method="post" >

$<$ div id='demographics'>

$<$ h1 $>$ Demographics $</$ h $1>$

$<\mathrm{p}>$

/>

Name: <input type='text' autocomplete="off" style="height: 29px; width: 200px" id="name" name='name' 


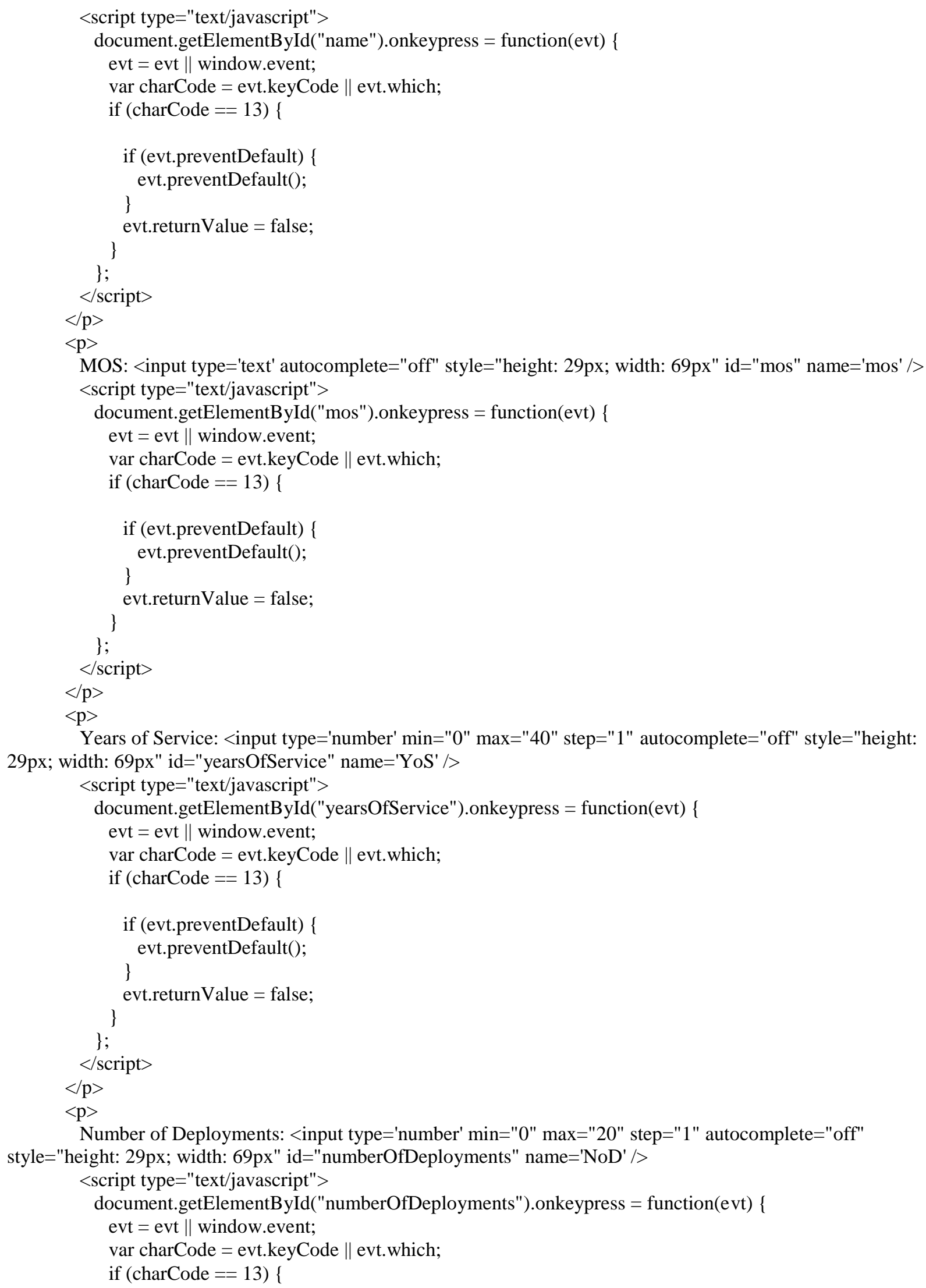




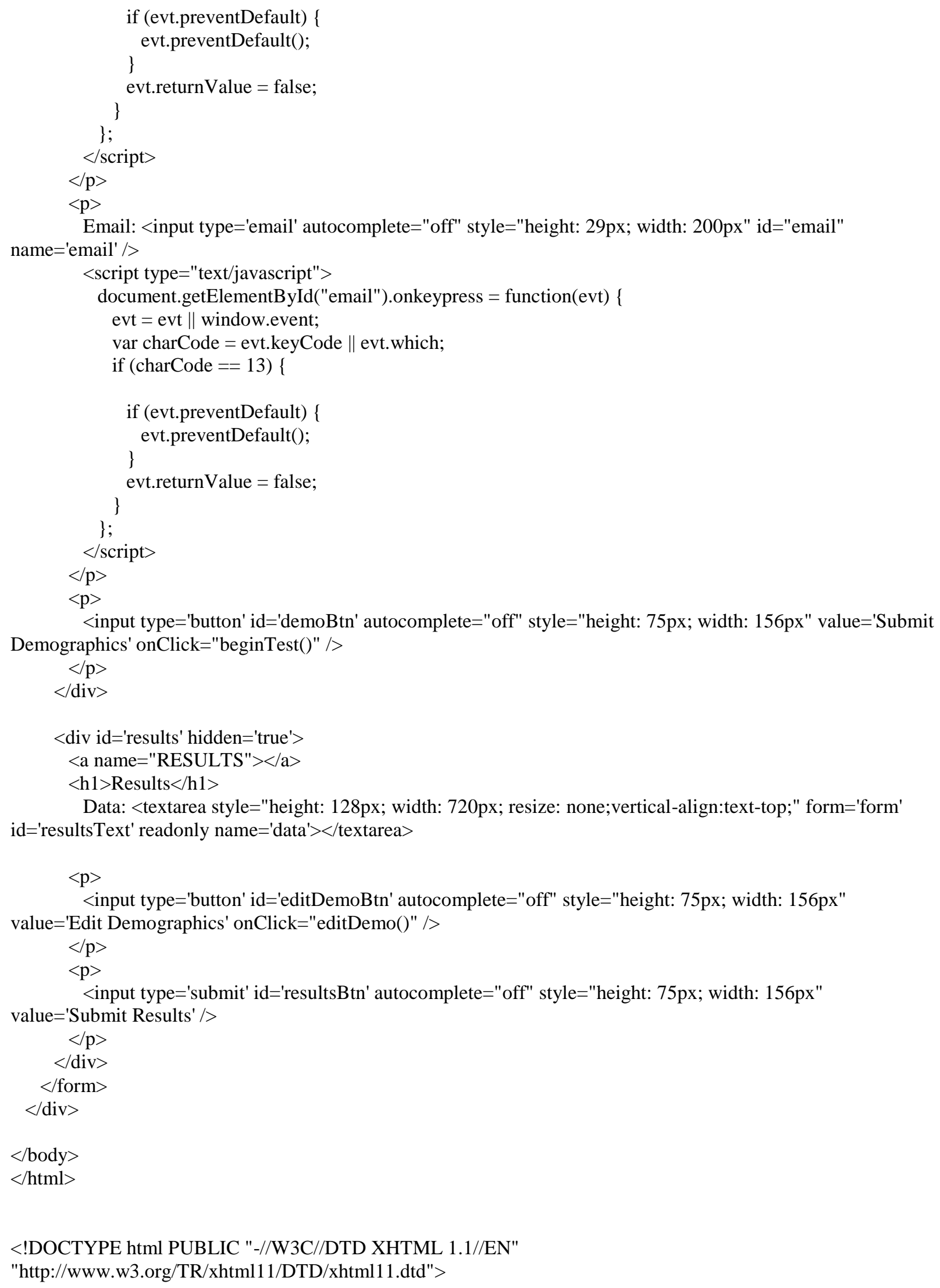




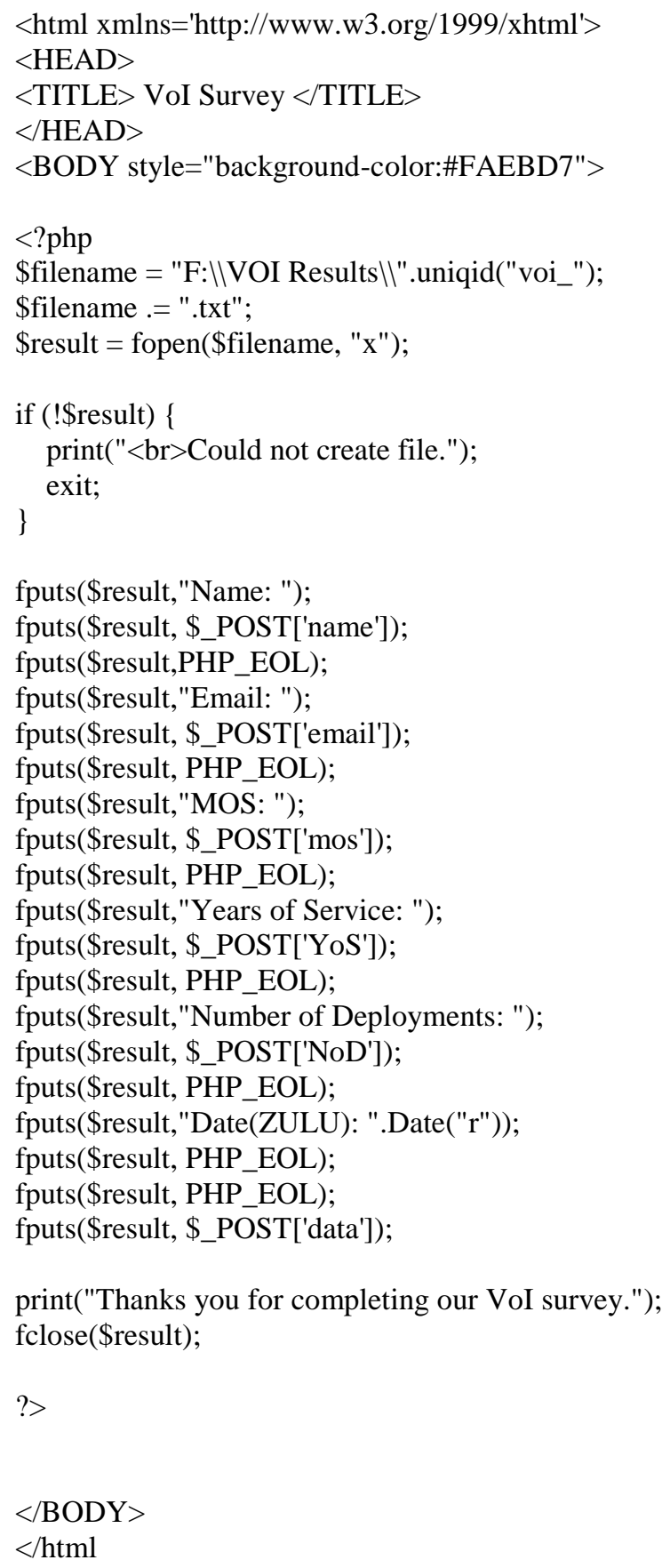




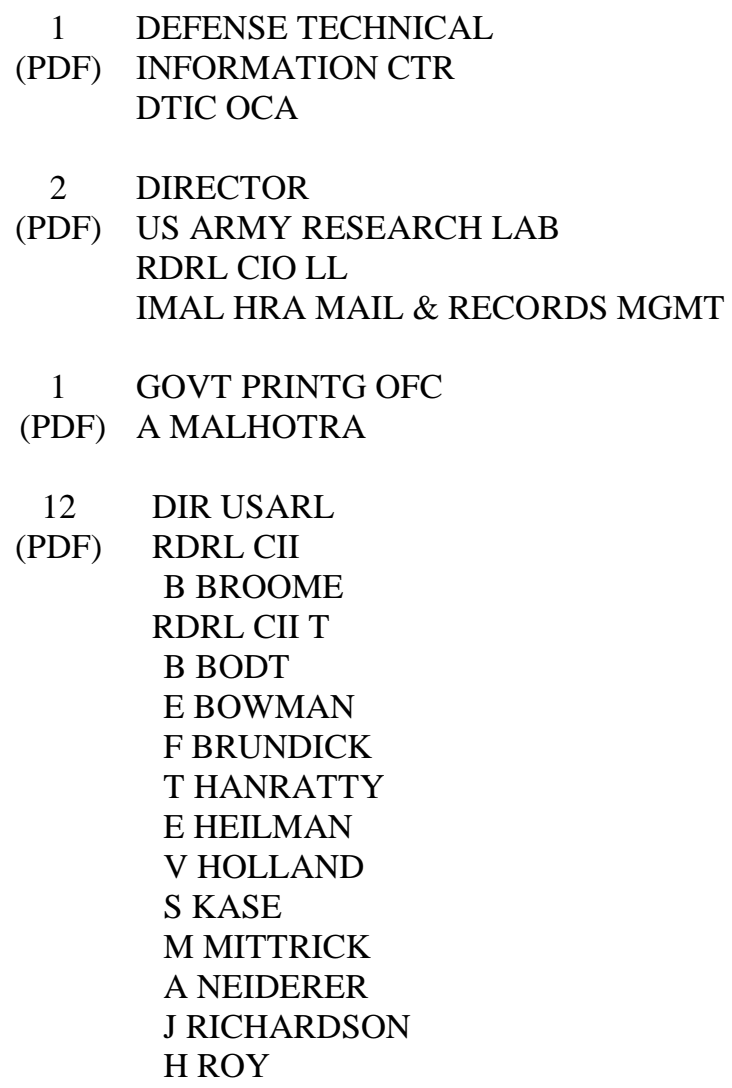

